\title{
Assemblage SiC/super alliage base nickel pour hautes températures
}

\author{
M.L. HATTALI ${ }^{1}$, S. VALETTE ${ }^{1}$, F. ROPITAL ${ }^{2}$, N. MESRATI ${ }^{3}$, D. TRÉHEUX ${ }^{1}$ \\ 1Laboratoire de Tribologie et Dynamique des Systèmes, UMR CNRS ECL ENISE ENSMSE 5513, École \\ Centrale de Lyon, 69134 Ecully cedex, France; \\ ${ }^{2}$ IFP, BP 3-69360 Solaize, France; \\ ${ }^{3}$ Laboratoire de Sciences et Génie des Matériaux, École Nationale Polytechnique d’Alger, 10, avenue de \\ Hassen Badi EL harrach Alger, Algerie; \\ hatlam69_2@hotmail.com
}

\begin{abstract}
Résumé
Ce travail porte sur l'étude de la réalisation des liaisons métal/céramique par thermocompression à l'état solide pour des applications à hautes températures $\left(>600^{\circ} \mathrm{C}\right)$. La liaison est obtenue entre le carbure de silicium (SiC) et un super alliage base nickel $\left(\right.$ HAYNES $^{\circledR} 214^{T M}$ ) en utilisant des joints métalliques purs tel que le nickel, le cuivre et l'argent. Le nickel et le cuivre forment des siliciures stables et à hauts points de fusion, mais ces produits de réaction sont très fragiles compte tenu de leurs sensibilité à "l'effet Pest ". Afin de minimiser cet effet nous avons ajouté du bore, réputé pour son action bénéfique, mais nous avons démontré que le bore ne peut à lui seul supprimer la fragilité des assemblages soumis de plus à des contraintes résiduelles. L'autre possibilité consiste à utiliser des dépôts minces d'argent ou des feuilles d'argent. Nous confirmons l'absence de réaction chimique à l'interface $\mathrm{Ag} / \mathrm{SiC}$ grâce à l'efficacité de la barrière d'argent à partir d'une certaine épaisseur. Cette méthode nous a permis de réaliser des assemblages avec une tenue mécanique de cisaillement (> $40 \mathrm{MPa})$.
\end{abstract}

\section{Mots clés:}

thermocompression, assemblage, métal/céramique, siliciures, SiC, super alliage base nickel

\section{INTRODUCTION}

Aujourd'hui, l'absence de solution technologique satisfaisante pour la réalisation d'assemblages $\mathrm{SiC} /$ métal [1-2] s'explique par la difficulté à résoudre essentiellement I'incompatibilité chimique (forte réactivité) et thermomécanique (notamment la différence de coefficient de dilatation thermique) qui existe entre la céramique et le métal.

De nombreux travaux ont étudié le mouillage mais aussi la réactivité entre $\mathrm{SiC}$ et de nombreux métaux tels que: $\mathrm{Al}$ [3], $\mathrm{Au}$ [4], $\mathrm{Cu}$ [5], Ni [5], Co [6]. Tous les métaux à l'exception de l'aluminium et l'argent sont à éviter, car ils conduisent à la formation de siliciures fragiles à bas point de fusion (proche de $900^{\circ} \mathrm{C}$ ). On distingue deux groupes de métaux en fonction de leur réactivité avec le SiC [7] :

1- les métaux réagissant uniquement avec le silicium qui conduisent à la formation de siliciures (Ni, Co, Fe...). Cette formation de siliciures s'accompagne d'une précipitation de carbone sous forme de feuillets graphitiques fragilisants pour l'assemblage [6].

2- les métaux présentant une réactivité vis-à-vis du silicium et du carbone $(\mathrm{Zr}, \mathrm{Ti}, \mathrm{Hf}, \mathrm{Mn} . .$.$) . Ces métaux peuvent$ conduire à une forte réactivité avec le $\mathrm{SiC}$ qui doit être contrôlée afin de sélectionner la nature des produits de réaction ainsi que leurs épaisseurs.

Ce problème du contrôle de la réactivité est d'autant plus important que l'assemblage doit fonctionner à haute température. La température élevée favorise la croissance des couches de réaction et provoque également une évolution de la chimie de la jonction non contrôlée, ce qui est dommageable à la tenue mécanique du joint. Le second point à considérer est la différence de comportement thermomécanique entre SiC et métal. Lors du refroidissement après thermocompression, la grande différence de coefficient de dilatation thermique entre les pièces à assembler induit des contraintes résiduelles d'origine thermomécaniques qui peuvent conduire à la rupture de la céramique, en raison de sa fragilité intrinsèque et de son module de Young élevé [8].

\section{PROCÉDURE EXPÉRIMENATLE}

Le carbure de silicium (ESK Ceramics type EKasic $\circledast$ F) est assemblé avec le superalliage par les différents joints métalliques $(\mathrm{Ni}, \mathrm{Cu}, \mathrm{Ag})$. Le rôle de ces joints consiste à la réduction des contraintes résiduelles générées par la différence de coefficients de dilatation thermique entre la céramique ( $\mathrm{SiC}$ ) et l'alliage (HAYNES ${ }^{\circledR} 214^{\mathrm{TM}}$ ). Leur choix est basé sur leurs hauts points de fusion. Quant au HAYNES $^{\circledR} 214^{T M}$, il est choisi dans cette étude pour son utilisation pour des applications technologiques aux hautes températures et dans les conditions sévères de l'industrie pétrochimiques. Tous les matériaux sont découpés en petite pièce parallélépipède $(15 \mathrm{~mm} \times 5 \mathrm{~mm} \times 5 \mathrm{~mm})$ pour assemblage. Les joints métalliques $\mathrm{Ni}, \mathrm{Cu}$ et $\mathrm{Ag}$ sont obtenues par laminage à froid jusqu'à $200 \mu \mathrm{m}$, avec des dimensions similaires à la céramique et l'alliage.

Dans certains expériences, et avant assemblage, un prédépôt type $\mathrm{Ni}_{0.93} \mathrm{~B}_{0.07}$ a était réaliser par le procédé JetMétal $^{\text {TM }}$ [9-10] sur la céramique. Le choix du prédépôt $\mathrm{Ni}_{0.93} \mathrm{~B}_{0.07}$ est basé sur la capacité du bore à améliorer les propriétés mécaniques des siliciures et d'éviter l'effet Pest

This is an Open Access article distributed under the terms of the Creative Commons Attribution-Noncommercial License (http://creativecommons.org/licenses/by-nc/3.0/), which permits unrestricted use, distribution, and reproduction in any noncommercial medium, provided the original work is properly cited. 
[11-13]. Le dépôt d'argent est utilisé comme barrière de diffusion.

La pureté des dépôts, joints métalliques, alliage et la céramique choisies dans notre étude sont :

- Dépôt NiB - (93\% Ni, $7 \%$ B ; $2 \mu \mathrm{m})$,

- Dépôt argent - $(99,9 \%$ de pureté ; $2 \mu \mathrm{m})$,

- Joint en nickel- (99,5\% de pureté ; $200 \mu$ m d'épaisseur),

- Joint en cuivre-(99,7\% de pureté ; $200 \mu \mathrm{m}$ d'épaisseur),

- Joint en argent- (99,9\% de pureté ; $200 \mu$ m d'épaisseur),

- HAYNES ${ }^{\circledR} 214^{\mathrm{TM}}$ - super-alliage base nickel (75\% Ni, 16

$\% \mathrm{Cr}, 4,5 \% \mathrm{Al}, 3 \% \mathrm{Fe})$,

- SiC - EKasic ${ }^{\circledR}-\mathrm{F}$ (> 99,2\% de pureté).

Avant assemblages, les surfaces de la céramique SiC (état de surface brut de frittage) subissent un polissage mécanique au feutre diamanté (14-1 $\mu \mathrm{m})$ afin d'obtenir une rugosité moyenne de $0,5 \mu \mathrm{m}$. Les surfaces des joints métalliques et l'alliage sont aussi poli mécaniquement en utilisant le papier abrasif à base de carbure de silicium (600), afin d'éliminer les couches d'oxydation. Toutes les surfaces sont nettoyées dans un bain d'ultra son d'acétone et d'éthanol pendant $10 \mathrm{~min}$.

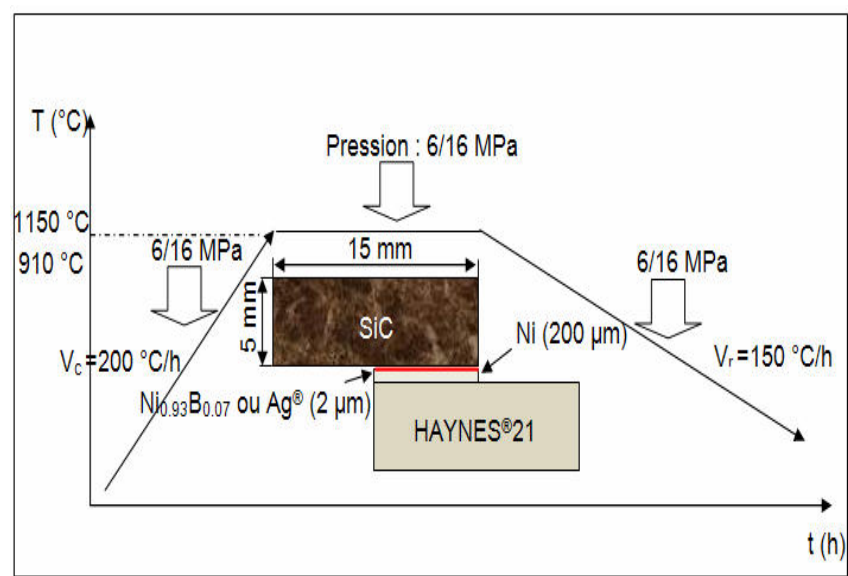

Figure 1: Schéma des géométries adoptées pour les assemblages, ainsi que le cycle thermique choisi pour la thermocompression.

Les assemblages sont réalisés dans un four-presse à une température inférieure aux points de fusion des joints métalliques et l'alliage, sous une pression de $16 \mathrm{MPa}$. La vitesse de chauffage est de $150{ }^{\circ} \mathrm{C} / \mathrm{h}$ alors que la vitesse du refroidissement est de $200{ }^{\circ} \mathrm{C} / \mathrm{h}$. l'assemblage est réalisé en vide primaire dynamique $\left(10^{-3} \mathrm{~Pa}\right)$ pendant tout le cycle de la réalisation 'Figure 1'.
Les conditions d'élaboration de différents assemblages sont mentionnées dans le tableau 1. Elles sont choisies en prenant considération de différentes études menées dans les assemblages alumina/Ni/alumina [14], alumine/Cu/alumine [15] et alumine/Ag/alumine [16].

La géométrie des assemblages est choisie en prenant en considération la possibilité de réalisation des essais mécaniques (essai de cisaillement) et les caractérisations physico-chimiques. Après assemblage, les zones interfaciale et fracturée dans l'assemblage $\mathrm{SiC} /$ metal sont observées et analysées par SEM/EDX et diffraction des rayons $X(X R D)$. Les essais de cisaillement sont conduits sur une machine type Instron. La vitesse de déplacement de la traverse est de $0.1 \mathrm{~mm} / \mathrm{min}$, et la contrainte à rupture est calculée en divisant la force à rupture de l'assemblage par la surface nominale de l'assemblage.

\section{RESULTATS ET DISCUSSION}

Durant notre étude et afin de limiter la réaction chimique entre l'alliage et la céramique, Nous avons procédé comme suit :

1- Utilisation d'un pré dépôt $\mathrm{Ni}_{0.93} \mathrm{~B}_{0.07}$ sur la céramique (2 $\mu \mathrm{m}$ d'épaisseur) afin de prévenir l'effet Pest et vérification de la réactivité de $\mathrm{SiC}$ avec le joint métallique 'Ni' et l'alliage HAYNES ${ }^{\circledR} 214^{\mathrm{TM}}$.

2- Utilisation d'un pré dépôt d'argent pur (2 $\mu \mathrm{m}$ d'épaisseur) comme barrière de diffusion.

3- Utilisation d'une feuille métallique d'argent pur $(200 \mu \mathrm{m}$ d'épaisseur) comme barrière de diffusion.

\section{1 $\mathrm{SiC} / \mathrm{Ni}_{0.93} \mathrm{~B}_{0.07} / \mathrm{Ni} /$ HAYNES $^{\mathrm{TM}} \mathbf{2 1 4}{ }^{\circledR}$}

Pour des températures supérieures à $700{ }^{\circ} \mathrm{C}$, le nickel en présence de $\mathrm{SiC}$ peut donner lieu à plusieurs réactions comme indiqué par le diagramme d'équilibre $\mathrm{Ni}-\mathrm{Si}$ [17] 'Figure 2'. Ces dernières dépendent de la concentration en silicium et de la température. Essentiellement des siliciures de nickel sont formés [5,17-20].

L'intérêt du prédépôt $\mathrm{Ni}_{0.93} \mathrm{~B}_{0.07}$ était d'améliorer la tenue mécanique des siliciures de $\mathrm{Ni}$ grâce à la présence du Bore [21]. Les examens métallographiques et par microscopie électronique à balayage après assemblage montrent qu'il existe une réactivité importante entre le nickel et le carbure de silicium ce qui conduit à la formation de plusieurs types de siliciures fragiles et cela malgré la présence du prédépôt $\mathrm{Ni}_{0.93} \mathrm{~B}_{0.07}$ 'Figure 3b'.

\begin{tabular}{|c|c|c|c|c|c|c|}
\hline Type d'assemblage & $\begin{array}{l}\text { Température } \\
\left({ }^{\circ} \mathrm{C}\right)\end{array}$ & $\begin{array}{l}\text { Pression } \\
(\mathrm{MPa})\end{array}$ & $\begin{array}{l}\begin{array}{l}\text { Temps } \\
\text { (h) }\end{array} \\
\end{array}$ & $\begin{array}{l}\begin{array}{l}\text { E }_{\text {feuille }} \\
(\mu \mathrm{m})\end{array} \\
\end{array}$ & $\begin{array}{l}\begin{array}{l}\text { edépôt } \\
(\mu \mathrm{m})\end{array} \\
\end{array}$ & Vide $(\mathrm{Pa})$ \\
\hline $\mathrm{SiC} / \mathrm{Ni}_{0.93} \mathrm{~B}_{0.07} / \mathrm{Ni}-\mathrm{HAYNES}{ }^{\circledR} 214^{\mathrm{TM}}$ & 1100 & 16 & 1 & 200 & 2 & $10^{-3}$ \\
\hline $\mathrm{SiC} / \mathrm{Ni}_{0.93} \mathrm{~B}_{0.07} / \mathrm{Cu}-\mathrm{HAYNES}^{\circledR} 214^{\mathrm{TM}}$ & 910 & 6 & 1 & 200 & 2 & $10^{-3}$ \\
\hline $\mathrm{SiC} / \mathrm{Ag} / \mathrm{HAYNES}^{\circledR} 214^{\mathrm{TM}}$ & 910 & 6 & 1 & 0 & 2 & $10^{-3}$ \\
\hline $\mathrm{SiC} / \mathrm{Ag} / \mathrm{HAYNES}^{\circledR} 214^{\mathrm{TM}}$ & 910 & 6 & 1 & 200 & 0 & $10^{-3}$ \\
\hline
\end{tabular}

Tableau 1: Conditions expérimentales de réalisations des assemblages $\mathrm{SiC} / \mathrm{HAYNES}^{\mathrm{TM}} 214^{\circledR}$ avec différents type de joints métalliques $(\mathrm{Ni}, \mathrm{Cu}, \mathrm{Ag})$ et de dépôts $\left(\mathrm{Ni}_{0.93} \mathrm{~B}_{0.07}\right.$ et $\left.\mathrm{Ag}\right)$. 


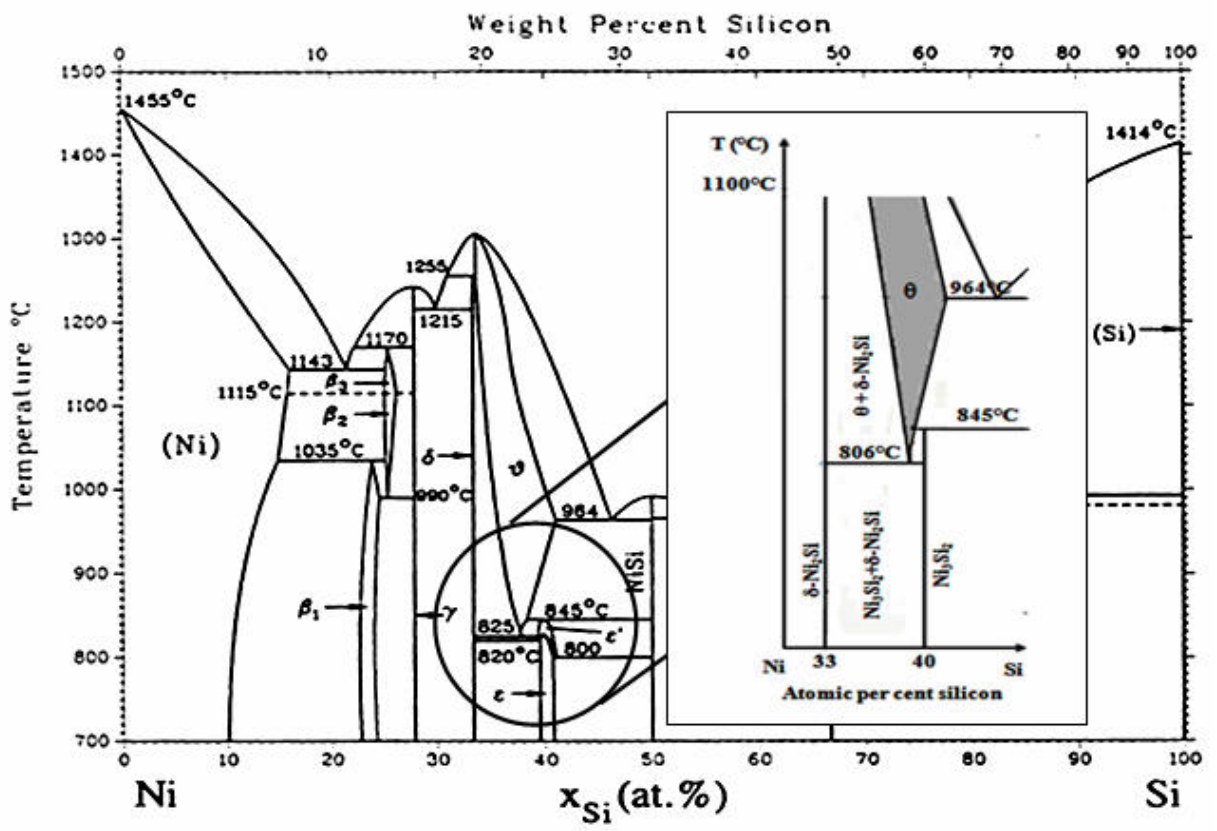

Figure 2 : Diagramme d'équilibre Ni-Si [17]

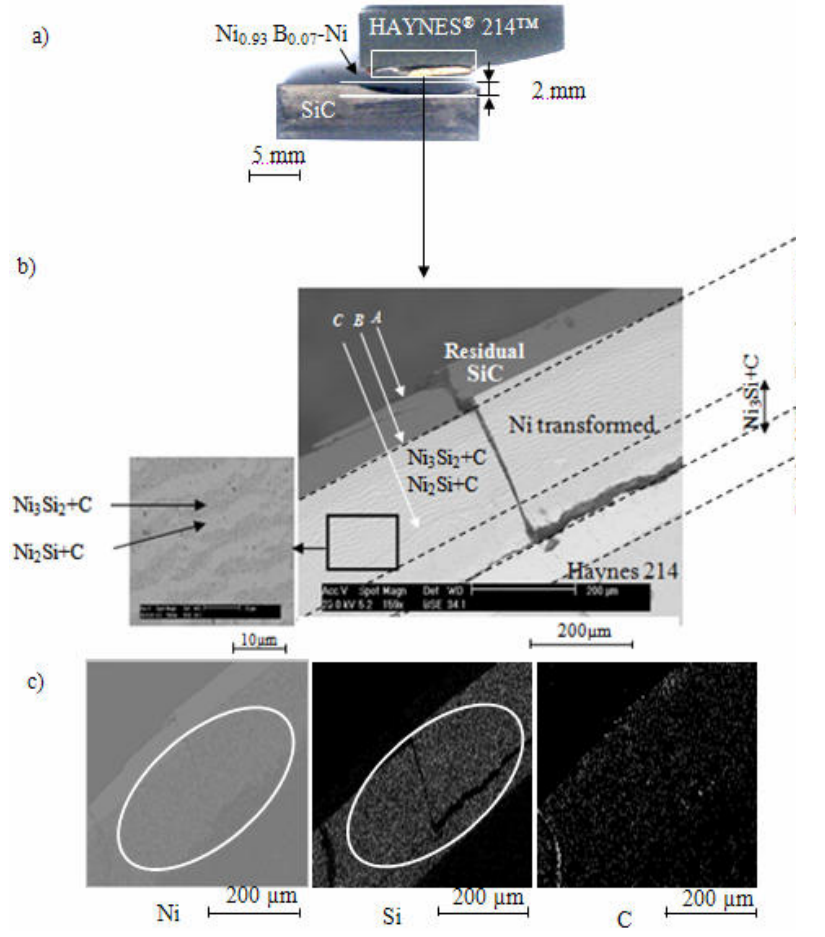

Figure 3 : Coupe transversale de la liaison $\mathrm{SiC} / \mathrm{Ni}_{0.93} \mathrm{~B}_{0.07}$ $\mathrm{Ni} / \mathrm{HAYNES}^{\mathrm{TM}} 214^{\circledR}\left(\mathrm{T}=1150{ }^{\circ} \mathrm{C}, \mathrm{t}=1\right.$ heure, $\left.\mathrm{P}=16 \mathrm{MPa}\right)$ a) macrographie optique de la zone de réaction, b) imagerie en électron rétrodiffusé (BSE) de la zone interfaciale. A, B, C sont les zones analysées par diffraction X (XRD) 'Figure 4', c) analyse par cartographie X de la zone assemblée.

La profondeur de la réaction varie entre 100 et $200 \mu \mathrm{m}$ avec une rupture de la céramique sur une profondeur de $2 \mathrm{~mm}$ 'Figure 3a et b', le métal d'apport initial (Nickel) a en majorité réagi avec le silicium. Ceci implique une activité thermodynamique du Nickel en présence du carbure de silicium et une cinétique de réaction importante.
Johnson et Rowcliffe [22] montrent que la différence de coefficient de dilatation thermique entre les céramiques et les métaux peut causer leurs ruptures à cause de la genèse des contraintes résiduelles. Dans notre cas, il est difficile de séparer le rôle des contraintes résiduelles de celui de la réactivité chimique à haute température que le bore ne supprime pas.

L'analyse quantitative par EDX nous a révélé la formation, dans la zone occupée initialement par le joint du nickel, de trois phases riches en $\mathrm{Si}$; elles contiennent respectivement 38.6, 31.2 et 26.4 at. \% Si 'Figure 3b'. Des analyses par cartographie $X$ des éléments $\mathrm{Ni}$, Si et $\mathrm{C}$, montrent que du graphite s'est formé et est distribué sur toute la profondeur de la zone de réaction 'Figure 3c'.

Des analyses complémentaires par diffraction des rayons $X$ après rupture de l'assemblage ont montré la présence de phases telles que $\mathrm{Ni}_{31} \mathrm{Si}_{12}$ (hexagonale), $\delta \mathrm{Ni}_{2} \mathrm{Si}$ (hexagonale) et le graphite. La troisième phase $\mathrm{BNi}_{3} \mathrm{~S}$ (cubique) n'a été détectée qu'après enlèvement totale du $\mathrm{SiC}$ résiduel par polissage mécanique 'Figure 4'. Les phases $\mathrm{Ni}_{31} \mathrm{Si}_{12}$ et $\delta \mathrm{Ni}_{2} \mathrm{Si}$ forment une structure bi-phasée de type eutectique, avec une épaisseur de $170 \mu \mathrm{m}$. En se référant au diagramme d'équilibre $\mathrm{Ni}-\mathrm{Si}$ 'Figure 1' et à nos observations nous pouvons supposer qu'au cours de la réalisation de l'assemblage les phases $\theta$ et $\beta \mathrm{Ni}_{3} \mathrm{Si}$ sont formées à une température de $1100{ }^{\circ} \mathrm{C}$, après refroidissement à $806{ }^{\circ} \mathrm{C}, \theta$ s'est décomposée par une réaction eutectoïde en $\mathrm{Ni}_{31} \mathrm{Si}_{12}+\delta \mathrm{Ni}_{2} \mathrm{Si}$. $\mathrm{Au}$ même moment, le graphite a été libéré et a précipité. $\beta \mathrm{Ni}_{3} \mathrm{Si}$ a une épaisseur de $70 \mu \mathrm{m}$ et est très fragile. La fragilité de cette phase est accompagnée par une rupture le long de l'interface $\mathrm{Ni} / \mathrm{Ni}_{3} \mathrm{Si}$ 'Figure 3 ' qui est attribué à la différence des coefficients de dilatation thermique entre le nickel et les siliciures formés. Des résultats analogues ont été obtenus par Schiepers et al. [19] après un traitement thermique du système $\mathrm{SiC} / \mathrm{Ni}$ à $850^{\circ} \mathrm{C}$ pendant $44 \mathrm{~h}$. 


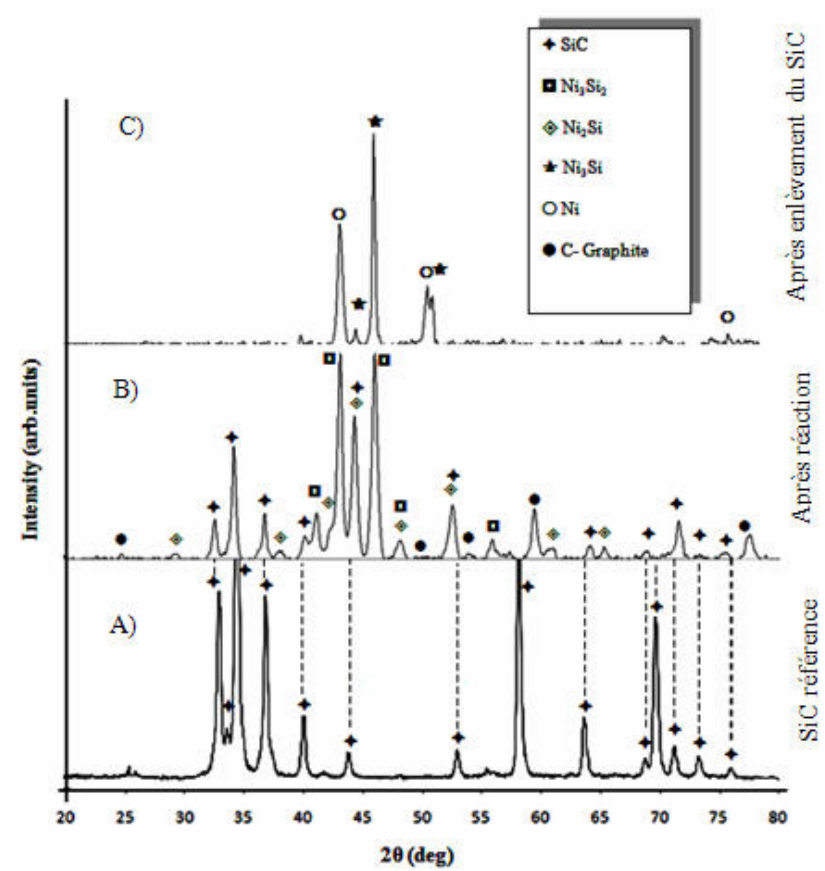

Figure 4 : Spectre de diffraction des rayons $X(\lambda \mathrm{Ka} \mathrm{Cu})$ de A) $\mathrm{SiC}$; B) Après rupture du $\mathrm{SiC}$ (zone de réaction) ; C) zone de réaction après enlèvement du $S i C$ résiduel. $(A, B$, C positions : voir Figure $2 b$ )

\section{$3.2 \mathrm{SiC} / \mathrm{Ni}_{0.93} \mathrm{~B}_{0.07} / \mathrm{Cu} / \mathrm{HAYNES}^{\mathrm{TM}} \mathbf{2 1 4}^{\circledR}$}

De même que le nickel, le cuivre est un métal très réactif vis-à-vis du carbure de silicium [17]. II forme plusieurs types de siliciures.

Dans ce cas aussi la liaison a échoué et pour les mêmes raisons que précédemment. Une forte réactivité du cuivre avec le SiC, cependant moins importante que dans le cas de $\mathrm{SiC} / \mathrm{Ni}_{0.93} \mathrm{~B}_{0.07}-\mathrm{Ni} / \mathrm{HAYNES}^{\mathrm{TM}} 214^{\circledR}$ et une rupture de la céramique sur une profondeur de $1 \mathrm{~mm}$. Des observations par microscopie électronique à balayage montrent l'existence de nouvelles phases formées au niveau de l'interface 'Figure 5'.

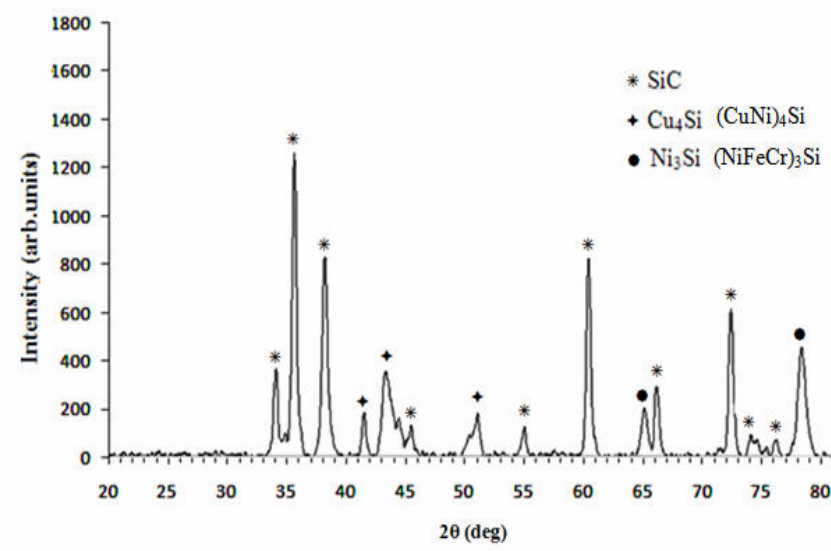

Figure 5 : Spectre de diffraction des rayons $X(\lambda \mathrm{Ka} \mathrm{Cu})$ de l'interface $\mathrm{SiC} / \mathrm{Cu}$ après rupture.

L'analyse EDX a confirmé l'hypothèse de la formation de ces siliciures. Notre analyse a mis en évidence la formation des siliciures quaternaire $\mathrm{Si}-\mathrm{FeCrNi}$ et ternaire $\mathrm{Si}-\mathrm{CuN}$ complexes. Des analyses complémentaires par diffraction des rayons $X$ après rupture de l'assemblage montrent que ces siliciures sont du type $\mathrm{Cu}_{4} \mathrm{Si} \quad\left((\mathrm{CuNi})_{4} \mathrm{Si}\right)$ et $\mathrm{Ni}_{3} \mathrm{Si}$ $\left((\mathrm{FeNiCr})_{3} \mathrm{Si}\right)$ 'Figure 5 et 6 '.

\section{3 $\mathrm{SiC} / \mathrm{Ag} / \mathrm{HAYNES}^{\mathrm{TM}} \mathbf{2 1 4 ^ { \circledR }}$}

\subsubsection{Utilisation d'un dépôt mince d'Ag}

Théoriquement, l'argent est un métal qui ne réagit pas avec le silicium et ne forme pas de siliciures [17]. Nous avons donc assemblé $\mathrm{SiC}$ avec le HAYNES ${ }^{\mathrm{TM}} 214^{\circledR}$ en utilisant tout d'abord un dépôt mince d'Ag $(\approx 2 \mu \mathrm{m})$ réalisé par la méthode JetMétal ${ }^{\circledR}$. L'analyse de l'assemblage $\mathrm{SiC} / \mathrm{Ag}^{\circledR} / \mathrm{HAYNES}^{\mathrm{TM}} 214^{\circledR}$ montre effectivement une faible réaction chimique partielle de $\mathrm{Ni}$ (élément principal de l'alliage) avec le SiC. Cette dernière a causé la rupture de la jonction à l'interface HAYNES ${ }^{\mathrm{TM}} 214^{\circledR} / \mathrm{SiC}$ compte tenu de l'épaisseur faible du dépôt d'argent qui a été traversé par le nickel provenant de l'alliage 'Figure 6'. a)

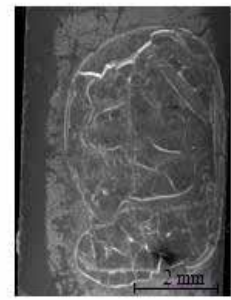

b)

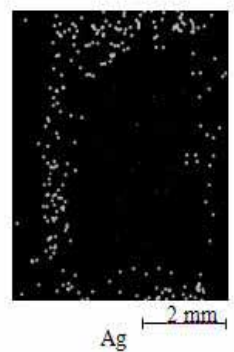

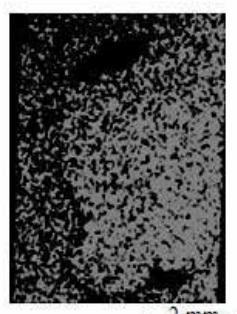

$\longmapsto 2 \mathrm{~mm}$
Figure 6 : Imagerie par microscopie électronique à balayage, après rupture, de l'assemblage $\mathrm{SiC} / \mathrm{Ag} / \mathrm{HAYNES}^{\mathrm{TM}} 214^{\circledR}$, réalisé avec un dépôt mince d'Ag $(\approx 2 \mu \mathrm{m})$. a) MEB, b) cartographie $X$.

a)
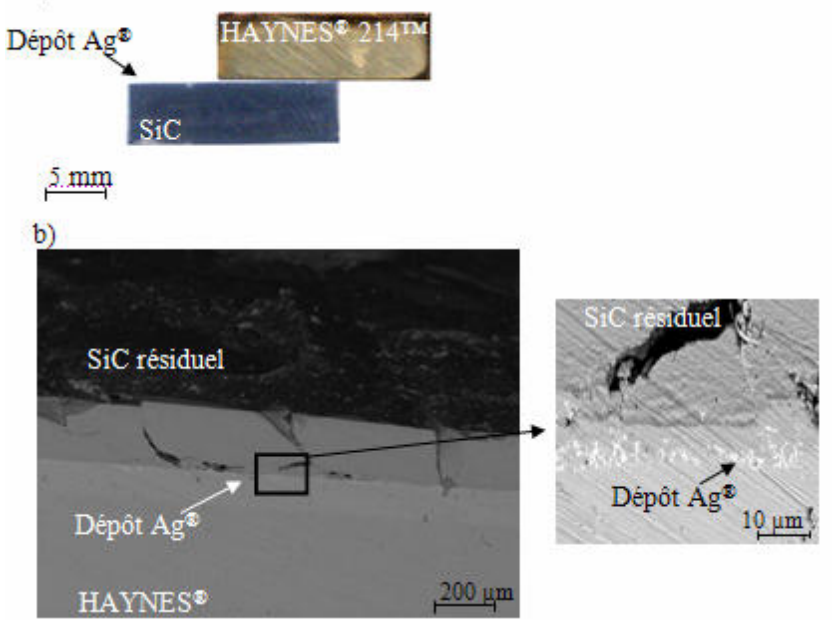

Figure 7: Coupe transversale de l'assemblage $\mathrm{SiC} / \mathrm{Ag} / \mathrm{HAYN} \mathrm{S}^{\mathrm{TM}} 214^{\circledR}$, a) Macrographie optique de l'assemblage. Micrographie par MEB, b) de l'interface $\mathrm{SiC} / \mathrm{Ag}$ montrant la non uniformité du dépôt $\mathrm{Ag}$ après assemblage.

En effet, l'analyse EDX de l'interface fracturée (la face de la céramique en contact avec l'alliage) montre des traces de nickel sur le SiC 'Figure 6b'. Deux raisons peuvent expliquer la présence du Ni sur le SiC. Premièrement, les observations par MEB après rupture de la zone interfaciale montrent que le dépôt d'Ag était discontinu 'Figure 7'. Par conséquent, le nickel a pu diffuser à travers ces discontinuités et ainsi réagir avec le SiC. II parait donc 
nécessaire d'augmenter l'épaisseur de ce dépôt. Deuxièmement, Tréheux et al [23] ont montré que les éléments qui ont une faible solubilité chimique, tel que le nickel et l'argent [24] $\left(D_{v(\mathrm{Ni} \rightarrow \mathrm{Ag})}\right.$ at $\left.910^{\circ} \mathrm{C}=5.10^{-13} \mathrm{~m}^{2} / \mathrm{s}\right)$, diffusent plus rapidement que ceux qui ont une forte solubilité. La diffusion du Ni à travers le dépôt $\mathrm{Ag}$ engendre la formation des siliciures de nickel fragiles, qui ont conduit à la rupture de l'interface. II apparaît après calcul et en utilisant la loi de la diffusion, $e=\sqrt{D_{V} t}$, (e, épaisseur de la diffusion, $D_{v}$, coefficient de diffusion et $t$, temps de diffusion) que le nickel peut diffuser sur une épaisseur de $43 \mu \mathrm{m}$ pour une durée de 1 heure à une température de $910{ }^{\circ} \mathrm{C}$ (conditions utilisées pour l'assemblage). Cependant, il faut noter qu'en utilisant ce dépôt mince d'argent $(\approx 2 \mu \mathrm{m})$, la réactivité a été déjà fortement réduite 'Figures 2 et 7'.Une utilisation d'un dépôt d'argent épais (> $50 \mu \mathrm{m})$ pourrait être une solution technologique intéressante.

\subsubsection{Utilisation d'une feuille d'Ag épaisse}

En se basant sur les résultats présentés ci-dessus, SiC est assemblé avec l'alliage de nickel (HAYNES ${ }^{T^{M}} 214^{\circledR}$ ) en utilisant une feuille d'Ag $(\approx 200 \mu \mathrm{m})$. L'analyse microstructurale d'une coupe transversale de la liaison par microscopie électronique à balayage (MEB) montre l'absence de réactivité chimique à cette échelle d'observation dans l'interface $\mathrm{Ag} / \mathrm{SiC}$. Les figures $8 \mathrm{~b}$ et $\mathrm{C}$ montrent une bonne adaptation de l'argent sur le SiC. Cependant, la présence d'oxydes et de porosités à l'interface Ag/HAYNES ${ }^{T M} 214^{\circledR}$ a été observée. On peut en conclure que le vide primaire utilisé lors de la réalisation des assemblages n'était pas suffisant pour éviter l'oxydation de l'alliage.

a)
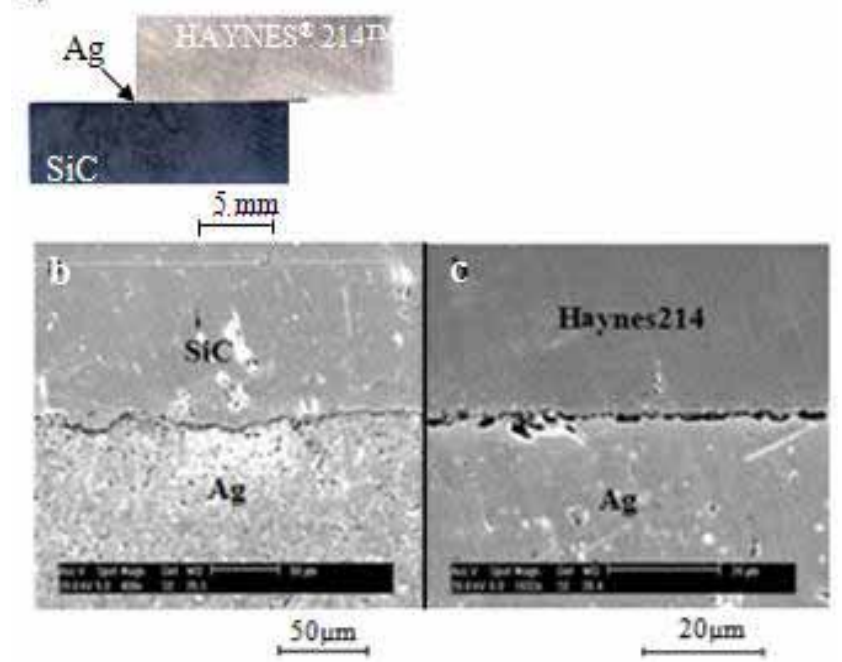

Figure 8: Coupe transversale de l'assemblage $\mathrm{SiC} / \mathrm{Ag} / \mathrm{HAYNES}{ }^{\mathrm{TM}} 214^{\circledR}$ a) Macrographie optique de la zone non réactive. Micrographie par MEB, b) de l'interface $\mathrm{SiC} / \mathrm{Ag}, \mathrm{c})$ de l'interface Ag/HAYNES ${ }^{\mathrm{TM}} 214^{\circledR}$.

La résistance mécanique des liaisons $\mathrm{SiC} / \mathrm{Ag} / \mathrm{HAYNES}{ }^{\mathrm{TM}} 214^{\circledR}$ en utilisant un joint d'Ag de 200 $\mu \mathrm{m}$ a été mesurée par un essai de cisaillement. Ce genre de test permet d'évaluer la qualité des assemblages du point de vue mécanique. Trois éprouvettes ont été testées. Les résultats obtenus donnent une contrainte moyenne de cisaillement de l'ordre de $25 \pm 6 \mathrm{MPa}$ avec une rupture adhésive à l'interface $\mathrm{Ag} / \mathrm{HAYNES}^{\mathrm{TM}} 214^{\circledR}$. Ce type de rupture est attribué à l'oxydation de l'alliage signalé précédemment et à la faible solubilité de l'Ag dans le nickel et le $\mathrm{Cr}$ (éléments constituant l'alliage HAYNES ${ }^{T M} 214^{\circledR}$ ). Afin d'améliorer le comportement mécanique de ces assemblages, nous avons réalisé d'autres types d'assemblages dans les mêmes conditions en utilisant des prédépôts $\mathrm{Ni}_{0.93} \mathrm{~B}_{0.07}$ sur l'alliage HAYNES ${ }^{\mathrm{TM}} 214^{\circledR}(\approx 2 \mu \mathrm{m})$. La contrainte moyenne de cisaillement obtenu est de l'ordre de $45 \pm 9 \mathrm{MPa}$ avec une rupture adhésive à l'interface $\mathrm{Ag} / \mathrm{HAYNES}{ }^{\mathrm{TM}} 214^{\circledR}$. Nos résultats montrent l'effet bénéfique de prédépôts $\mathrm{Ni}_{0.93} \mathrm{~B}_{0.07}$ sur l'amélioration du comportement mécanique de l'assemblage essentiellement à l'interface Ag/HAYNES ${ }^{T M} 214^{\circledR}$ qui a permis de limiter l'oxydation de l'alliage HAYNES ${ }^{\mathrm{TM}} 214^{\circledR}$.

Les calculs de diffusion précédemment effectués dans le cas d'un dépôt mince d'Ag sont appliqués maintenant pour une épaisseur de joint d'Ag de $200 \mu \mathrm{m}$. A partir de ces calculs, on peut estimer la durée de vie des assemblages en d'autres termes le temps qu'il faut pour que le Ni diffuse à travers l'Ag pour venir réagir avec SiC. Le tableau 2 donne les résultats obtenus en fonction de la température d'utilisation.

\begin{tabular}{|c|l|l|l|}
\hline $\begin{array}{c}\text { Type } \\
\text { d'assemblage }\end{array}$ & $\begin{array}{l}\text { Coefficient } \\
\text { de diffusion } \\
{[23]\left(\mathrm{m}^{2} / \mathrm{s}\right)}\end{array}$ & $\mathrm{T}\left({ }^{\circ} \mathrm{C}\right)$ & \multicolumn{1}{|c|}{$\begin{array}{c}\text { Durée } \\
\text { de vie } \\
(\mathrm{h})\end{array}$} \\
\hline & $5 \times 10^{-17}$ & 600 & $>200 \times 10^{3}$ \\
SiC/Ag/HAYNES & $10^{-15}$ & 700 & $>10 \times 10^{3}$ \\
& $10^{-14}$ & 800 & $>1000$ \\
& $5 \times 10^{-13}$ & 900 & $>22$ \\
\hline
\end{tabular}

Tableau 2: Estimation théorique de la durée de vie de l'assemblage $\mathrm{SiC} / \mathrm{Ag} / \mathrm{HAYNES}^{\circledR} 214^{\mathrm{TM}}$ en fonction de la température pour un joint en $\mathrm{Ag}$ de $200 \mu \mathrm{m}$ d'épaisseur.

A partir de ces calculs on peut en conclure que la solution proposée $(\mathrm{Ag} \approx 200 \mu \mathrm{m})$ est une solution technologique intéressante pour des applications industrielles devant travailler entre 600 et $800{ }^{\circ} \mathrm{C}$. Les temps estimés sont respectivement de $200000 \mathrm{~h}$ et $1000 \mathrm{~h}$.

\section{CONCLUSION}

En dépit de son intérêt technologique pour desapplications thermomécaniques, le carbure de silicium pose de nombreux problèmes lorsqu'il est lié à un métal pour des températures supérieures à $600^{\circ} \mathrm{C}$, en raison de sa forte réactivité avec les différents métaux qui entraîne la formation de siliciures fragiles. Ainsi, pour résoudre ce problème, différents assemblages SiC/HAYNES ${ }^{\circledR} 214^{\text {TM }}$ ont été réalisées, en utilisant; un dépôt $\mathrm{Ni}_{0.93} \mathrm{~B}_{0.07}$, un dépôt $\mathrm{Ag}$, et une feuille d'Ag. Dans le premier cas, nous avons démontré que l'ajout de bore par l'intermédiaire d'un dépôt $\mathrm{Ni}_{0.93} \mathrm{~B}_{0.07}$ n'est pas suffisant pour éviter la fragilité des siliciures qui développent de fortes contraintes résiduelles à l'interface au cours du processus de fabrication (essentiellement au cours du refroidissement). Dans le second cas, l'utilisation d'un dépôt $\mathrm{Ag}(\approx 2 \mu \mathrm{m})$ permet d'éviter de manière significative la réaction du carbure de silicium avec le nickel. Dans cette logique une nouvelle approche d'assemblage entre le carbure de silicium et l'alliage à base de nickel pour les applications à hautes températures $\left(600{ }^{\circ} \mathrm{C}<\mathrm{T}<800{ }^{\circ} \mathrm{C}\right)$ à l'aide d'un joint épais d'Ag $(\approx 200 \mu \mathrm{m})$ a été explorée. La capacité à former une liaison forte et à éviter les réactions entre le $\mathrm{SiC}$ et le nickel a été démontrée. L'utilisation d'une feuille d'argent $(\approx 200 \mu \mathrm{m})$ entre le $\mathrm{SiC}$ et l'alliage (HAYNES $^{\circledR} 214^{\mathrm{TM}}$ ) améliore la résistance au cisaillement mécanique à $45 \pm 9$ $\mathrm{MPa}$, ce qui peut laisser espérer des applications industrielles à $700^{\circ} \mathrm{C}$ pendant plus de 10000 heures. 


\section{REFERENCES}

[1].Lipa, M, "TPX Workshop on plasma facing components", in development and fabrication of new generation of CFC-brazed plasma facing componement to installed in Tore Supra, 1995.

[2].Doceul, L, "18th symp on fusion technology", in conceptual design of high heat flux toroidal pumped limiter for Tore Supra, Germany, 1994.

[3].Projet Brite-Euram AMBRA-Production of high temperature resistance joints between engineering ceramics and metals by active metals brazing-Final Technical Report, Brite-Euram Program, Contract BRE2CT92-0350-October 1997.

[4].Drevet, B, Kalogerolpolou.S, Eustathopoulos. N (1993). Acta.Mater, 41 pp.3119-3126.

[5].Rado, C, Drevet. B, Eustathopoulos. N (2000). Acta.Mater, 48 pp 4483-4491.

[6].Tang, W, Zheng.Z, Ding.H, Jin.Z (2002). Mater.Chem.Phys, 74 pp 258-264.

[7].Lamy, $M$, Thèse institut national polytechnique de Grenoble, 2000.

[8].Treheux, D, Lourdin, P, Mbongo, B, Juve, D (1994). Scripta Metall Mater, 31(8), pp 1055-1060.

[9].Stremsdoerfer, G., Procédé direct de revêtement métallique par projection de solution aqueuses sur tout type de substrat, Brevet 97 06-834, publication n FR276396 1997.

[10]. Stremsdoerfer, G., Ghanem, F., Saikali, Y. and Fares Karim, A. (2003), J. Mater. Sci, 38, pp 3291-3299.

[11].Berztiss, R. R., Cerchiara, E. A, Gulbransen pettit, F. S, Meier, G. H., J.Mater.Sci. Eng. A, 1992, A155, 165-172.
[12]. Aoki, K, Izumi. O. (1974), J. Jpn. Inst. Met., 1979, 43, pp 358-359.

[13]. Aoki, K, Izumi. O, (1979), J. Jpn. Inst. Met., 43, pp 1190-1196.

[14].Lourdin, P., Juvé, D, Tréheux, D, (1996), J.Eur. Ceram. Soc, 16(7), 745-752.

[15].Kara-Slimane, A., Mbongo, B. Tréheux, D., (1999). J. Adhesion Sci.Technol, 13, pp 35-48.

[16].Serier, B. Tréheux, D. (1993). Acta Metall. Mater, 41(2), pp 369-374.

[17]. Hansen, M. and Anderko, K., Constitution of Binary Alloys, vol. 1. McGraw-Hill, New York, 1958, pp. 10391041.

[18]. Meyer, M. K., Kramer, M. J. and Akinca, M., 1996, Adv. Mater., 8(1), pp 85-88.

[19]. Schiepers, R. C. J., Van Beek, J. A., van Loo, F. J. J. and de With, G., 1993, J. Eur. Ceram. Soc.,11, pp 211218.

[20].Schiepers, R. C. J., van Loo, F. J. J, de With, G., 1988, J. Amer. Ceram. Soc.,71(6), pp 248-287.

[21].Xiong,H., 2007, Matere.Lett., 16, pp 4662-4665

[22].Johonson, S., Rowcliffe, D., J. Amer. Ceram. Soc., 68(9), pp 85-88.

[23].Tréheux, D., Heurtel, A. and Guiraldeng, P.,1976, Acta Met., 24, pp 503-509.

[24].Hirone, T., Miura, S. and Susuoka, T., 1961. J. Phys.Soc. Japan, 16, pp 2456-2459. 